\title{
Focusing Attention on the Health Aspects of Foods Changes Value Signals in vmPFC and Improves Dietary Choice
}

\author{
Todd A. Hare, Jonathan Malmaud, and Antonio Rangel \\ Computation and Neural Systems and Humanities and Social Science Divisions, California Institute of Technology, Pasadena, California 91125
}

\begin{abstract}
Attention is thought to play a key role in the computation of stimulus values at the time of choice, which suggests that attention manipulations could be used to improve decision-making in domains where self-control lapses are pervasive. We used an fMRI food choice task with non-dieting human subjects to investigate whether exogenous cues that direct attention to the healthiness of foods could improve dietary choices. Behaviorally, we found that subjects made healthier choices in the presence of health cues. In parallel, stimulus value signals in ventromedial prefrontal cortex were more responsive to the healthiness of foods in the presence of health cues, and this effect was modulated by activity in regions of dorsolateral prefrontal cortex. These findings suggest that the neural mechanisms used in successful self-control can be activated by exogenous attention cues, and provide insights into the processes through which behavioral therapies and public policies could facilitate self-control.
\end{abstract}

\section{Introduction}

We often make choices between complex options that differ in multiple attributes. For example, foods differ in their short-term nutritional and long-term health consequences. Studies from psychology and behavioral economics suggest that the stimulus values of complex multi-attribute stimuli (e.g., taste, healthiness, size, and packaging for foods) are computed by assigning values to the individual attributes and integrating them (Bettman et al., 1998). Furthermore, behavioral studies (Shimojo et al., 2003; Armel et al., 2008; Krajbich et al., 2010), as well as psychological models of decision-making (Busemeyer and Towsend, 1993; Roe et al., 2001; Rieskamp et al., 2006), suggest that the way attributes are integrated depends on how the individual's attention is deployed among the various features. These integrated stimulus value signals are then compared with make a choice (Montague and Berns, 2002; Wallis, 2007; Rangel et al., 2008; Kable and Glimcher, 2009; Rushworth et al., 2009; Schoenbaum et al., 2009; Rangel and Hare, 2010). This model of value computation is consistent with recent fMRI studies of intertemporal and risky choices showing that neural activity correlates with stimulus value signals composed of integrated temporal, risk, and reward features of the stimuli (Kable and Glimcher, 2007; Tom et al., 2007; Levy et al., 2010). The model also implies that optimal decisionmaking requires assigning values to stimuli in a way that properly reflects their relative contribution to the benefits (or experienced utility) they generate.

An important source of self-control failures could be a tendency of the valuation circuitry to overweight short-term (e.g.,

Received Dec. 8, 2010; revised April 28, 2011; accepted June 15, 2011.

Author contributions: T.A.H. designed research; T.A.H. and J.M. performed research; T.A.H., J.M., and A.R. analyzed data; T.A.H. and A.R. wrote the paper.

This work was supported by National Science Foundation Grant 0851408 to T.A.H. and A.R.

The authors declare no conflict of interest.

Correspondence should be addressed to Todd Hare, Computation and Neural Systems and Humanities and Social Science Divisions, California Institute of Technology, HSS 228-77, Pasadena, CA 91125-7700. E-mail: thare@hss.caltech.edu.

DOI:10.1523/JNEUROSCI.6383-10.2011

Copyright $\odot 2011$ the authors $\quad 0270-6474 / 11 / 3111077-11 \$ 15.00 / 0$ taste) relative to long-term features (e.g., healthiness) (Liberman and Trope, 2008). Such a relative weighting scheme would result in choices that are excessively driven by short-term considerations at the expense of potential long-term costs. Consistent with this view, a previous fMRI study of dietary choice found that value signals encoded in ventral medial prefrontal cortex (vmPFC) overweighted short- compared with long-term features in individuals who failed to exercise self-control (Hare et al., 2009). That study also found that vmPFC activity correlated with the overall stimulus value participants placed on a food at the time of choice, regardless of their degree of self-control. However, there was an important difference between successful and non-successful self-controllers: in self-controllers, the vmPFC signal reflected both taste and health considerations; and in nonselfcontrollers, it reflected only taste. Moreover, self-controllers activated a region of dorsolateral prefrontal cortex (dlPFC) that appeared to modulate vmPFC to incorporate health information. These findings suggest that the difference between value signals (and subsequent choices) computed by dietary self-controllers and nonself-controllers depends on their ability to incorporate health information into vmPFC value signals.

These results give rise to two questions. First, can exogenous cues that draw attention to health features of foods induce healthier choices? Second, do such manipulations of attention operate by increasing the degree to which dlPFC modulates vmPFC so that its activity reflects healthiness? In other words, can simple exogenous cues activate the processes that successful self-controllers seem to activate endogenously?

\section{Materials and Methods}

Subjects. Data from 33 subjects ( 10 male; mean age: 24.8 years, SD, 5.1 years) are presented here. Subjects were screened to insure that they were not currently following any specific diet or seeking to lose weight for any reason. All subjects were right-handed, healthy, had normal or correctedto-normal vision, had no history of psychiatric diagnoses or neurological or metabolic illnesses, and were not taking medications that interfered 
with the performance of fMRI. Five additional subjects participated in the experiment, but were excluded from analysis due to excessive within-run head motion $(>2 \mathrm{~mm}$ in any direction) or low signal-to-noise levels in ventral regions of their functional scans based on visual analysis. The review board of the California Institute of Technology (Pasadena, CA) approved the study.

Stimuli. Subjects rated and made decisions on 180 different food items, including junk foods (e.g., chips and candy bars) and healthier snacks (e.g., apples and broccoli). The foods were presented to the subjects using highresolution color pictures ( $72 \mathrm{dpi}$ ). The stimulus presentation and response recording was controlled through Matlab using the Psychophysics Toolbox extensions (Brainard, 1997). The visual stimuli were presented using video goggles.

Task. Subjects were instructed not to eat for $3 \mathrm{~h}$ before the experiment. On every trial, subjects were shown a picture of one of the food items and were given up to $3 \mathrm{~s}$ to indicate whether they wanted to eat that food at the end of the experiment. The picture of the food dis-

appeared as soon as a response was entered. They indicated their responses using a four-point scale: Strong No, No, Yes, and Strong Yes. At the end of the experiment, one of the trials was randomly selected to count. If the subject said "Yes" or "Strong Yes" for that item, they had to eat that food at that time. If they said "No" or "Strong No," then they did not have to eat the food. In either case, they were asked to stay in the lab for $30 \mathrm{~min}$ after the experiment. Note that because only one random trial was selected to count, the optimal strategy for subjects is to treat each decision as if it were the only one. Trials were separated by a variable intertrial interval (ITI) of $4-6 \mathrm{~s}$. To control trial length, $3 \mathrm{~s}$ minus the reaction time were added to the ITI after each trial.

Subjects were asked to make choices under three different attention conditions. In the health condition (HC), they were asked to consider the healthiness of the food before choosing. In the taste condition (TC), they were asked to consider the taste of the food before choosing. In the control or natural condition (NC), they were asked to consider whatever features of the food came naturally to mind. Critically, the instructions emphasized that subjects should always make the decision that they prefer, regardless of the attention condition. The attention condition was kept constant for 10 trials at a time, and the beginning of a new block was announced through a $5 \mathrm{~s}$ screen (Fig. 1). After the instructions, subjects completed 180 trials in the scanner, 60 in each instruction condition. Each food was shown only once and the order of foods and blocks was fully randomized for each subject.

After leaving the scanner, subjects completed two self-paced computer tasks. In one, they rated each food for its perceived taste (scale: very untasty, untasty, tasty, very tasty). In the second task, they rated each food for its perceived healthiness (scale: very unhealthy, unhealthy, healthy, very healthy). The order in which each subject completed these tasks was counterbalanced across subjects.

Note that a few features of the task played an important role in the analyses. First, we recruited subjects who were not currently seeking to lose weight or restricting their dietary intake in any specific way. This was done because we were interested in investigating the impact of the attention cues on individuals without preexisting dietary goals. Second, subjects indicated their choices using a four-point scale. This is useful because it allows us to measure simultaneously their behavioral choice (eat vs not) and the stimulus value assigned to the item at the time of decision (strong no $=-2$, no $=-1$, yes $=1$, and strong yes $=2$ ). Note that all subjects reported their decisions using the entire decision scale. Third, the postscanning health and taste ratings provide independent measures of the taste and health features of each food stimulus that were only modestly correlated with each other (cross-subject mean: $r=0.320$,
$\mathrm{SD}=0.315)$. These ratings are essential for investigating the role that health and taste information play in the computation of stimulus value at both the behavioral and neural levels. Fourth, only one of the decisions is implemented at random at the end of the experiment. As a result, the subject's optimal strategy is to treat each decision as if it were the only one.

Behavioral data analysis. The behavioral data were analyzed in two different ways. First, we estimated a mixed-effects linear regression model of stimulus values on health ratings (HR), taste ratings (TR), a dummy for $\mathrm{HC}$, a regressor for $\mathrm{HC}$ interacted with $\mathrm{HR}\left(\mathrm{HR}^{\star} \mathrm{HC}\right)$, a regressor for $\mathrm{HC}$ interacted with $\mathrm{TR}\left(\mathrm{TR}^{\star} \mathrm{HC}\right)$, a dummy for taste consideration condition (TC), a regressor for TC interacted with HR $\left(\mathrm{HR}^{\star} \mathrm{TC}\right)$, and a regressor for TC interacted with $\mathrm{TR}\left(\mathrm{TR}^{\star} \mathrm{TC}\right)$. The stimulus values were measured with the four-point scale used at the time of decision (strong no $=-2$, no $=-1$, yes $=1$, strong yes $=2$ ). Second, the choices in each of the three conditions were binned into four categories (untasty and unhealthy, untasty and healthy, tasty and unhealthy, tasty and healthy). The probability of choosing to eat (given by a yes or strong yes response) foods in each bin is shown in Figure $2 b$.

Five subjects made responses to the ratings and decisions using a fivepoint scale $(-2,-1,0,1,2)$. The behavioral results are unchanged when including or excluding these five subjects and we therefore pooled them with the rest of the sample.

MRI data acquisition. T2*-weighted echoplanar images (EPI) were acquired using a Siemens 3.0 Tesla Trio MRI scanner with an eightchannel phased array coil. To optimize functional sensitivity in the orbitofrontal cortex, we used an oblique acquisition orientation of $30^{\circ}$ above the anterior-posterior commissure line (Deichmann et al., 2003). Each volume was comprised of 44 axial slices collected in an interleaved ascending manner. Functional data were collected in three sessions. The length of each session varied slightly and was on average 208 volumes $(9.5$ $\mathrm{min})$. The EPI parameters were as follows: echo time, $30 \mathrm{~ms}$; field of view, $192 \mathrm{~mm}$; interleaved acquisition; in-plane resolution, $3 \times 3 \mathrm{~mm}$; slice thickness, $3 \mathrm{~mm}$; and repetition time, $2.75 \mathrm{~s}$. Whole-brain highresolution T1-weighted structural scans $(1 \times 1 \times 1 \mathrm{~mm})$ were acquired from all subjects and coregistered with their mean EPI images and averaged together to permit anatomical localization of the functional activations at the group level.

fMRI data preprocessing. Image analysis was performed using SPM8 (Wellcome Department of Imaging Neuroscience, Institute of Neurology, London, UK). Images were corrected for slice acquisition time within each volume, motion corrected with realignment to the middle volume, spatially normalized to the standard Montreal Neurological In- 


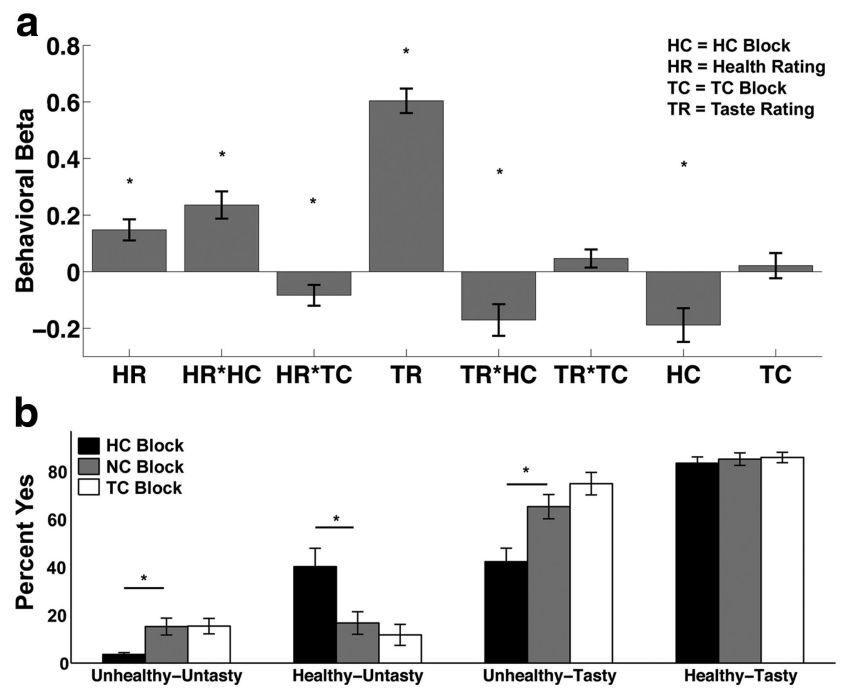

Figure 2. Behavioral results. $\boldsymbol{a}$, The bar graph shows the mean beta weight across subjects for each regressor in the behavioral GLM. Positive beta weights indicate that a factor increased the likelihood of eating the food item and negative beta weights indicate that it decreased the likelihood of eating the food. Asterisks identify regressors that are significantly different from zero at $p<0.05 . \boldsymbol{b}$, Bar graph showing the percentage of the time subjects responded "yes" to eat the food as a function of taste-health combination and block type. Asterisks identify bins where health trials were significantly different from natural trials at $p<0.05$. Error bars represent SEM in both graphs.

stitute EPI template, and spatially smoothed using a Gaussian kernel with a full-width-at-half-maximum of $8 \mathrm{~mm}$. Intensity normalization and high-pass temporal filtering (using a filter width of $128 \mathrm{~s}$ ) were also applied to the data.

Basic fMRI analyses. We analyzed the fMRI data using two main GLMs of BOLD responses with first-order autoregression.

GLM 1 had the following six regressors of interest: (1) an indicator for any decision event with a duration equal to the reaction time for the trial, (2) the decision event indicator modulated by the stimulus value behavioral measure, (3) an indicator for health blocks modeled as the period from the offset of the health consideration instructions to the onset of the next instruction set, (4) an indicator for taste blocks modeled as the period from the offset of the taste consideration instructions to the onset of the next instruction set, (5) an indicator for an attention instruction (5 s duration), and (6) missed trials where no response was made within the $3 \mathrm{~s}$ time window. Session constants and motion parameters were included in the model as regressors of no interest. For this GLM, we calculated the following first-level single-subject contrasts: (1) decision events modulated by stimulus value, (2) health consideration block effect, and (3) taste consideration block effect. Note that, given the specification of our GLM, contrasts 2 and 3 measure the change in average activity with respect to the natural control condition blocks. Here and below, secondlevel group contrasts were computed using one-sample $t$ tests on the single-subject contrasts.

GLM 2, which parallels the linear regression analysis for the behavioral data, had 11 regressors: (1) indicator for all decision events with a duration equal to reaction time, (2) all decisions events modulated by health ratings, (3) all decision events modulated by taste ratings, (4) indicator function for health blocks, (5) indicator for decisions during health blocks modulated by taste ratings, (6) indicator for decisions during health blocks modulated by health ratings, (7) indicator function for taste blocks, (8) indicator function for decisions during taste blocks modulated by taste ratings, (9) indicator function for decisions during taste blocks modulated by health ratings, (10) indicator for missed trials, and (11) indicator for block instruction screens. For this GLM, we calculated the following first-level single-subject contrasts: (1) decision events modulated by taste ratings, (2) decision events modulated by health ratings, (3) decision events during health blocks modulated by taste ratings, (4) decision events during health blocks modulated by
Table 1. Regions positively correlated with stimulus value independent of attention condition

\begin{tabular}{|c|c|c|c|c|c|c|c|}
\hline Region & BA & Side & $\begin{array}{l}\text { Cluster } \\
\text { size }\end{array}$ & $x$ & $y$ & Z & Peak Z score \\
\hline Posterior cingulate & 31 & $\mathrm{R}$ & 67 & 3 & -34 & 37 & $3.97^{*}$ \\
\hline $\begin{array}{l}\text { vmPFC (anterior cingulate/ } \\
\text { orbitofrontal cortex) }\end{array}$ & $32 / 10$ & L & 267 & -9 & 50 & 4 & $3.85^{*}$ \\
\hline
\end{tabular}

Height threshold, $t=3.37$; extent threshold, 20 voxels $(3 \times 3 \times 3 \mathrm{~mm})$. R, Right; L, left.

*The activation survives whole-brain correction $(p<0.05)$ for multiple comparisons at the cluster level (height threshold, $t=3.37$; extent, 56 voxels).

Table 2. Regions positively correlated with health ratings in NC blocks

\begin{tabular}{|c|c|c|c|c|c|c|c|}
\hline Region & $\mathrm{BA}$ & Side & $\begin{array}{l}\text { Cluster } \\
\text { size }\end{array}$ & $x$ & $y$ & $z$ & Peak Z score \\
\hline \multirow{2}{*}{$\begin{array}{l}\text { vmPFC (anterior cingulate/ } \\
\text { orbitofrontal cortex) }\end{array}$} & $10 / 11$ & \multirow[t]{2}{*}{$\mathrm{L}$} & \multirow[t]{2}{*}{196} & \multirow[t]{2}{*}{-6} & \multirow[t]{2}{*}{41} & \multirow[t]{2}{*}{-14} & \multirow[t]{2}{*}{$3.93^{*}$} \\
\hline & $25 / 32$ & & & & & & \\
\hline Middle frontal gyrus & 6 & $\mathrm{~L}$ & 28 & -30 & 5 & 70 & 3.83 \\
\hline Superior frontal gyrus & 8 & $\mathrm{~L}$ & 189 & -27 & 20 & 52 & $3.70^{*}$ \\
\hline Superior frontal gyrus & 8 & $\mathrm{R}$ & 34 & 18 & 44 & 55 & 3.61 \\
\hline Middle temporal gyrus & 37 & $\mathrm{~L}$ & 40 & -54 & -52 & -11 & 3.54 \\
\hline Middle/inferior frontal gyrus ${ }^{\ddagger}$ & $9 / 44$ & $\mathrm{~L}$ & 64 & -45 & 11 & 22 & $3.45^{\dagger}$ \\
\hline Cuneus & 18 & $\mathrm{R}$ & 52 & 0 & -91 & 16 & 3.37 \\
\hline Posterior cingulate & 31 & $\mathrm{R}$ & 140 & 0 & -64 & 25 & 3.36 \\
\hline Inferior frontal gyrus & 46 & $\mathrm{~L}$ & 36 & -48 & 32 & 10 & 3.33 \\
\hline Superior parietal lobule & $19 / 39$ & $\mathrm{~L}$ & 70 & -42 & -67 & 31 & 3.17 \\
\hline
\end{tabular}

Height threshold, $t=2.74$; extent threshold, 20 voxels $(3 \times 3 \times 3 \mathrm{~mm})$. L, Left; $\mathrm{R}$, right.

*The activation survives whole-brain correction $(p<0.05)$ for multiple comparisons at the cluster level (height threshold, $t=2.74$; extent 179 voxels).

${ }^{\dagger}$ Peak for ROl included in the conjunction in Figure $5 b$.

${ }^{\ddagger}$ Referred to as dIPFC-M in DCM figures.

Table 3. Regions positively correlated with taste ratings in NC blocks

\begin{tabular}{llllllll}
\hline \multirow{2}{*}{ Region } & BA & Side & size & $x$ & $y$ & $z$ & PeakZscore \\
\hline vmPFC (anterior cingulate/ & $10 / 32$ & $\mathrm{~L}$ & 286 & -12 & 44 & 1 & $4.37^{*}$ \\
$\quad$ orbitofrontal cortex) & & & & & & & \\
Thalamus/parahippocampal gyrus & 30 & $\mathrm{~L}$ & 97 & -24 & -31 & 4 & 4.31 \\
Parahippocampal gyrus & 30 & $\mathrm{R}$ & 60 & 21 & -37 & 1 & 4.15 \\
Posterior cingulate cortex & $31 / 23$ & $\mathrm{R}$ & 170 & 9 & -31 & 34 & $3.90^{*}$ \\
Cuneus & 18 & $\mathrm{R}$ & 37 & 15 & -85 & 19 & 3.73 \\
Posterior cingulate cortex & 30 & $\mathrm{~L}$ & 33 & -15 & -55 & 4 & 3.55 \\
Anterior cingulate cortex & 24 & $\mathrm{~L}$ & 34 & -3 & 14 & 22 & 3.55 \\
Precuneus & 7 & $\mathrm{~L}$ & 35 & -9 & -70 & 40 & 3.23 \\
\hline
\end{tabular}

Height threshold, $t=2.74$; extent threshold, 20 voxels $(3 \times 3 \times 3 \mathrm{~mm})$. L, Left; $\mathrm{R}$, right.

${ }^{*}$ The activation survives whole-brain correction $(p<0.05)$ for multiple comparisons at the cluster level (height threshold, $t=2.74$; extent, 137 voxels).

health ratings, (5) decision events during taste blocks modulated by health ratings, and (6) decision events during taste blocks modulated by taste ratings. Note that in this specification of the model, regressors 2 and 3 measure the impact of taste and health ratings during the $\mathrm{NC}$, and regressors $2+5$ and $2+6$ measure the impact of the ratings during $\mathrm{HC}$ and TC, respectively.

For every GLM, we performed whole-brain corrections for multiple comparisons at the cluster level. The details of corrections for each contrast are listed in Tables 1-6. The individual contrasts comprising the conjunction analysis for areas reflecting $\mathrm{HR}$ and showing increased coupling with vmPFC at the time of choice were computed at $p<0.005$ with an extent threshold of 20 voxels given the more stringent requirement that an area show both effects. Figures 3-5 show the resulting statistical parametric maps overlaid on a normalized structural image averaged across subjects.

Post hoc analyses. We estimated an additional GLM (GLM 3) for the purpose of a planned post hoc analysis. This third GLM included eight regressors: (1) decision events in natural blocks (with a duration equal to the reaction time), (2) natural block decisions modulated by stimulus 
Table 4. Regions more active in health blocks than natural blocks

\begin{tabular}{|c|c|c|c|c|c|c|c|}
\hline Region & $\mathrm{BA}$ & Side & Cluster size & $x$ & $y$ & $z$ & Peak Z score \\
\hline Inferior frontal gyrus & $47 / 10 / 46$ & L & 661 & -42 & 41 & 1 & $6.09 *$ \\
\hline Inferior parietal lobule & 40 & L & 435 & -48 & -64 & 55 & $5.34^{*}$ \\
\hline Middle temporal gyrus & 21 & $\mathrm{R}$ & 110 & 66 & -40 & -14 & $5.21^{*}$ \\
\hline Inferior parietal lobule & 40 & $\mathrm{R}$ & $86 s$ & 54 & -61 & 46 & $5.20 *$ \\
\hline Superior frontal gyrus & $8 / 9$ & $\mathrm{~L}$ & 421 & -18 & 56 & 31 & $4.97^{*}$ \\
\hline Middle temporal gyrus & $21 / 22$ & L & 240 & -54 & -31 & -2 & $4.42^{*}$ \\
\hline Middle frontal gyrus & 10 & $\mathrm{R}$ & 40 & 39 & 50 & 13 & 3.9 \\
\hline Middle frontal gyrus $^{\dagger}$ & $9 / 8$ & $\mathrm{~L}$ & 72 & -36 & 8 & 43 & $3.84^{*}$ \\
\hline Superior frontal gyrus & 9 & $\mathrm{R}$ & 22 & 12 & 47 & 37 & 3.8 \\
\hline
\end{tabular}

Height threshold, $t=3.37$; extent threshold, 20 voxels $(3 \times 3 \times 3 \mathrm{~mm})$. L, Left; $\mathrm{R}$, right.

*The activation survives whole-brain correction $(p<0.05)$ for multiple comparisons at the cluster level (height threshold, $t=3.37$; extent, 67 voxels).

${ }^{\dagger}$ Referred to as DLPFC-U in DCM figures.

Table 5. Regions more active in taste blocks than natural blocks

\begin{tabular}{llllllll}
\hline Region & BA & Side & Cluster size & $x$ & $y$ & $Z$ & Z score \\
\hline Inferior parietal lobule & 40 & $\mathrm{~L}$ & 152 & -48 & -64 & 52 & $4.99^{*}$ \\
Inferior parietal lobule & 40 & $\mathrm{R}$ & 89 & 51 & -61 & 46 & $4.27^{*}$ \\
Superior frontal gyrus & $9 / 10$ & $\mathrm{~L}$ & 30 & -15 & 59 & 31 & 3.79 \\
Middle temporal gyrus & 21 & $\mathrm{~L}$ & 44 & -57 & -28 & -14 & 3.57 \\
\hline
\end{tabular}

Height threshold, $t=3.37$; extent threshold, 20 voxels $(3 \times 3 \times 3 \mathrm{~mm})$. L, Left; $\mathrm{R}$, right.

*The activation survives whole-brain correction $(p<0.05)$ for multiple comparisons at the cluster level (height threshold, $t=3.37$; extent, 56 voxels).

Table 6. Regions showing increased coupling (PPI) with vmPFC at the time of decision in all blocks

\begin{tabular}{lrllllll}
\hline Region & BA & Side & Cluster size & $x$ & $y$ & $z$ & Z score \\
\hline Precentral/inferior frontal gyrus & 9 & $\mathrm{~L}$ & 25 & -48 & 2 & 31 & $3.03^{\dagger}$ \\
Parietal lobule & 40 & $\mathrm{R}$ & 24 & 60 & -37 & 58 & 2.92 \\
\hline
\end{tabular}

Height threshold, $t=2.74$; extent threshold, 20 voxels $(3 \times 3 \times 3 \mathrm{~mm})$. L, Left; $R$, right.

${ }^{\dagger}$ Peak for $\mathrm{ROI}$ included in the conjunction in Figure $5 b$.

value, (3) decisions in health blocks, (4) health block decisions modulated by stimulus value, (5) decisions in taste blocks, (6) taste block decisions modulated by stimulus value, (7) instruction times, and (8) missed trials where no response was made within the $3 \mathrm{~s}$ time window. Session constants and motion parameters were included in the model as regressors of no interest. The contrasts for regressors 2,4 , and 6 were used to create the bar graph shown in Figure $3 b$. Beta values for each subject were averaged within a $4 \mathrm{~mm}$ sphere centered on the subject's individual peak within the group peak for the contrast of all decisions modulated by stimulus value in GLM 1 (Fig. $3 a$ ).

In a second post hoc analysis, we compared the association between beta coefficients in the behavioral regression and the corresponding coefficients from vmPFC for the fMRI regression (referred to as GLM 2, above). Our primary interest was in the $\mathrm{HR}^{\star} \mathrm{HC}$ coefficient, which measured the change in the effect of health ratings on stimulus values during $\mathrm{HC}$ blocks, but all eight coefficients were tested for completeness. A single beta coefficient from the behavioral regression served as the dependent measure while regressors 2-9 from GLM 2 were the explanatory variables in a between-subjects robust regression analysis (bisquare estimator). One example of the regression equation used is as follows:

$\mathrm{HR}{ }^{*} \mathrm{HC}_{\mathrm{B}}=\beta_{0}+\beta_{1}{ }^{*} \mathrm{HR}_{\mathrm{N}}+\beta_{2}{ }^{*} \mathrm{HR}{ }^{*} \mathrm{HC}_{\mathrm{N}}+\beta_{3}{ }^{*} \mathrm{HR}{ }^{*} \mathrm{TC}_{\mathrm{N}}+$ $\beta_{4} * \mathrm{TR}_{\mathrm{N}}+\beta_{5} * \mathrm{TR}^{\star} \mathrm{HC}_{\mathrm{N}}+\beta_{6} * \mathrm{TR} * \mathrm{TC}_{\mathrm{N}}+\beta_{7} * \mathrm{HC}+\beta_{8} * \mathrm{TC}$,

where the subscript $\mathrm{B}$ signifies a behavioral decision coefficient and $\mathrm{N}$ signifies a neural coefficient. In this example, we would be specifically interested in $\beta_{2}$ because it measures the association between the matching decision and neural coefficients. Values for the neural coefficients for each subject were averaged within a $4 \mathrm{~mm}$ sphere centered on the subject's individual peak within the group vmPFC ROI for the contrast of all decisions modulated by stimulus value from GLM 1 described above. Note that GLM 1 does not contain parameteric regressors for health or taste ratings or their interactions with condition. a
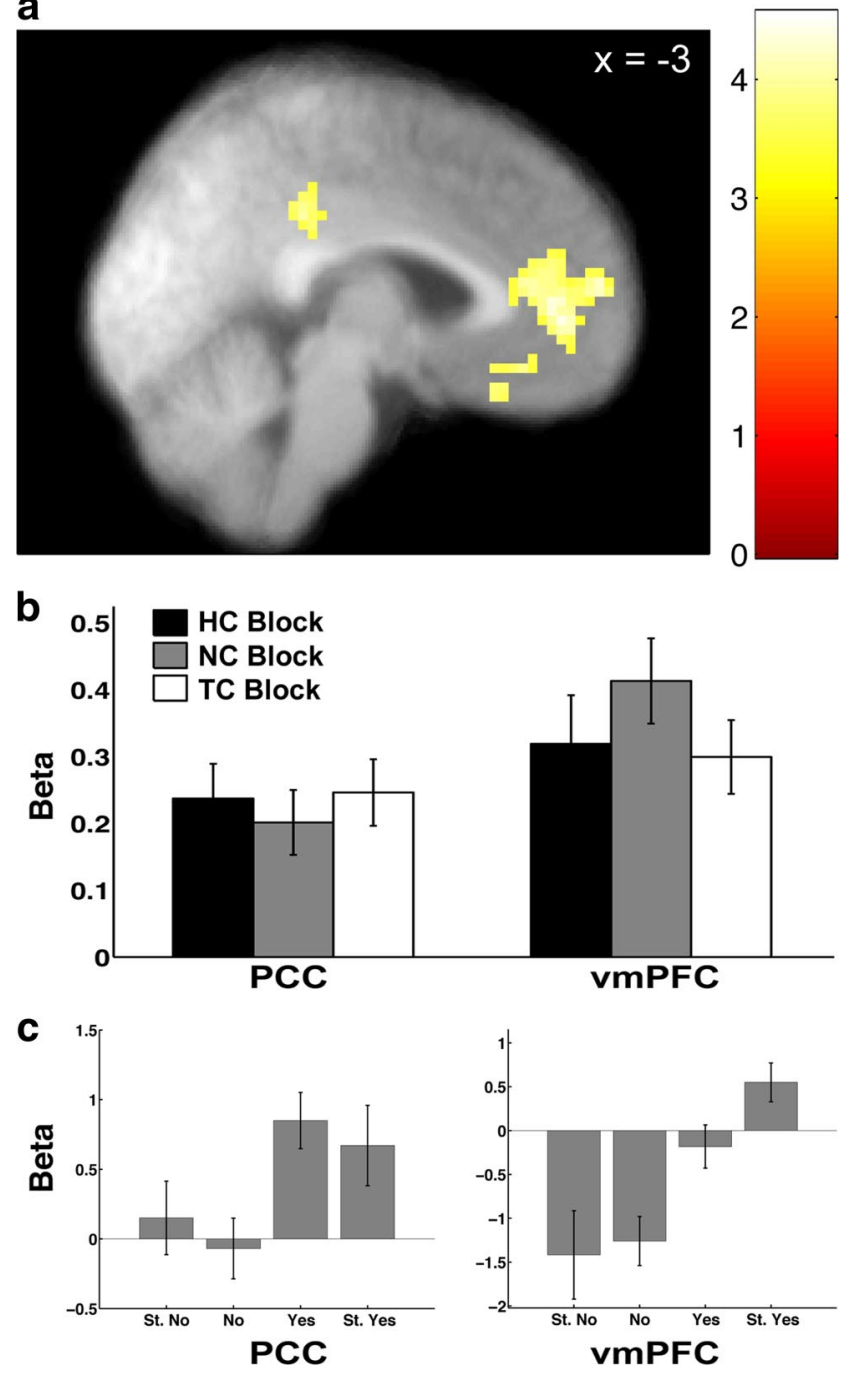

Figure 3. Activity correlated with stimulus value at the time of choice. $\boldsymbol{a}$, Activity in vmPFC and PCC correlated with stimulus value at $p<0.05$, corrected. $\boldsymbol{b}$, Beta plots showing that the correlation with stimulus value in vmPFC and PCC did not differ between blocks. Error bars represent SEM. c, Beta plots displaying the amount of activity in vMPFC and PCC at each level of stimulus value for visualization purposes only.

Psychophysiological interactions. We performed a psychophysiological interaction (PPI) analysis to identify regions exhibiting an increase in correlation with the vmPFC at the time of decision-making. This was done in three steps.

First, for each individual, we extracted the BOLD time-series from the voxel within a $5 \mathrm{~mm}$ sphere surrounding the individual activation peak for the contrast of all decisions modulated by stimulus value from GLM 1 , within the group mask of the vmPFC shown in Figure 3. Variance associated with the six motion regressors was removed from the extracted time-series. The time courses were then deconvolved based on the model for the canonical hemodynamic response to construct a time series of neural activity in the vmPFC following the procedures outlined in Gitelman et al. (2003).

Second, for every subject, we estimated a GLM that included the following three regressors as well as motion parameters: (1) an interaction between neural activity in the vmPFC and the indicator for decision time convolved with the canonical hemodynamic response function (HRF), (2) an indicator for decisions events (with a duration equal to the reaction time) convolved with the canonical HRF, and (3) the original BOLD eigenvariate from the vmPFC (i.e., the first principal component of timeseries from the voxels within the $5 \mathrm{~mm}$ sphere). Single subject contrasts were calculated following estimation of the GLM. 

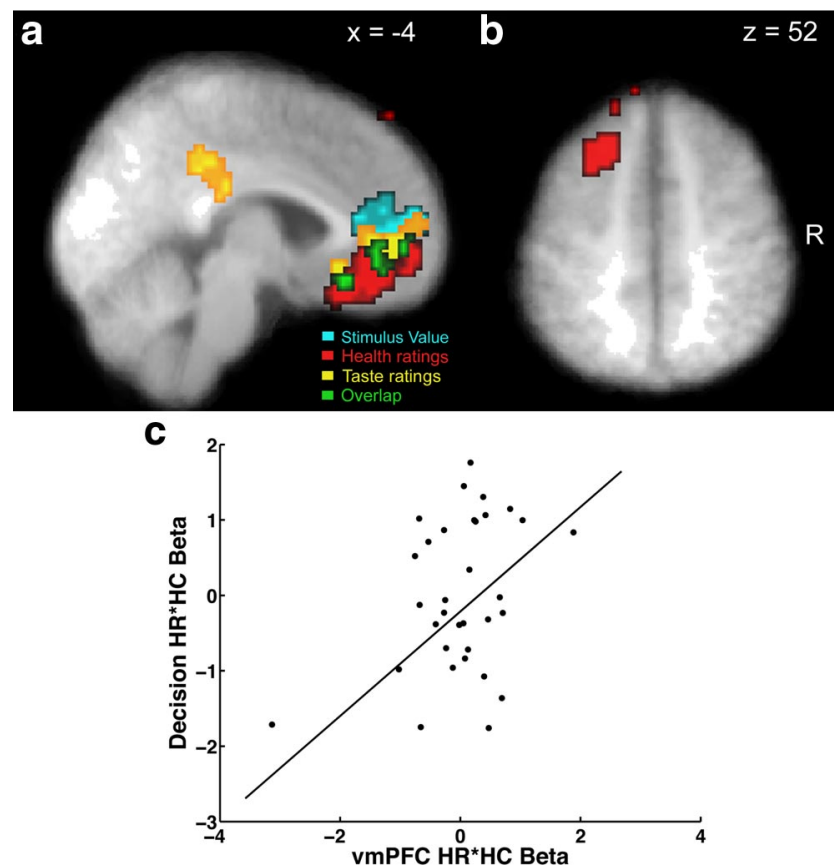

Figure 4. Activity in vmPFC correlated with both health and taste ratings at the time of decision. Voxels in red are correlated with the parametric regressor for $\mathrm{HR}$, and those in yellow are correlated with the parametric regressor for TR. Voxels in cyan are correlated with the parametric regressor for stimulus value. All three contrasts are thresholded at $p<0.05$, corrected. The conjunction of these three contrasts is shown in green. $\boldsymbol{a}$, Sagittal section showing vmPFC and PCC correlated with HR and TR. $\boldsymbol{b}$, Axial section showing a region of left dIPFC correlated with HR. $c$, The scatterplot shows the relationship between the beta coefficient for the interaction of health ratings and health consideration blocks from the behavioral regression for decisions and the same coefficient for vmPFC activity after removing the variance associated with the other vmPFC coefficients in the second fMRI GLM analysis. Each point denotes an individual. The line represents the fit from a robust regression analysis using a bisquare estimator.

Third, second-level group contrasts were calculated based on the single-subject contrast values using one-sample $t$ tests.

Dynamic causal modeling. We examined the functional connectivity between regions using nonlinear dynamic causal modeling (DCM) (Stephan et al., 2008). The analysis was performed in several steps.

First, for every individual, we extracted four activation time courses from functional masks of vmPFC (Fig. 3), dlPFC ummodulated by HRs (dlPFC-U; BA 8/9) (Fig. 5a), inferior frontal gyrus (IFG; BA 47/46) (Fig. $5 a$ ), and dlPFC modulated by HRs (dlPFC-M; BA 9) (Fig. $5 b$ ). We created the functional masks for the first three regions using an individual voxel threshold of $p<0.001$ and the extent threshold specified in the corresponding contrast tables. The functional mask for dlPFC-M was created from all voxels showing the conjunction described in Figure $5 b$. The time course for each subject was averaged within from a $5 \mathrm{~mm}$ sphere centered on the individual subject peak within the group ROI for the appropriate contrast.

Second, we specified 30 different models of potential connectivity between the four ROIs. These models included the fully connected model and all possible combinations of connectivity created by removing one to four bidirectional connections from the full model. Four driving inputs of BOLD activity were specified for all models: (1) input to vmPFC at the time of choice modulated by taste ratings, (2) input to dIPFC-M at the time of choice modulated by health ratings, (3) input to dIPFC-U and IFG at the time of choice (unmodulated), and (4) input into dlPFC-U and IFG for the duration of the health blocks. In addition, all models allowed modulation in the coupling strength of all existing parameters at the time of choice.

Third, we identified the most likely model using the Bayesian model selection (BMS) method developed by Stephan et al. (2009). Briefly, this technique treats the models as random variables and computes a distribution of the probabilities for all models under consideration. The prob-
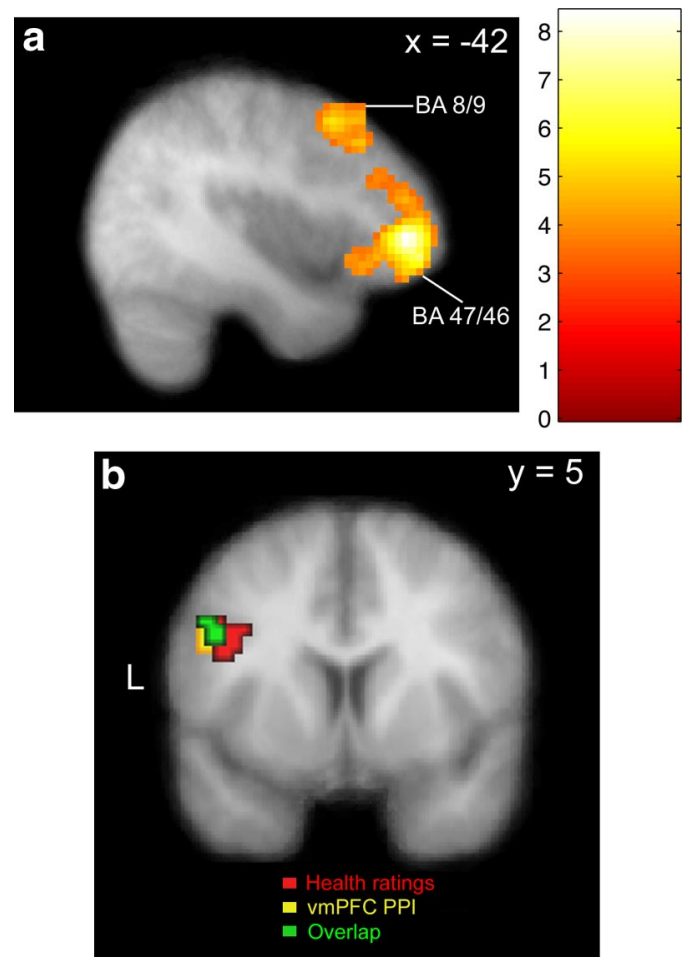

Figure 5. Potential effectors of the attention-induced changes. $\boldsymbol{a}$, The statistical parametric map shows regions of lateral prefrontal cortex in BA 8/9 and 46/47 where activity increases during $\mathrm{HC}$ blocks ( $p<0.05$, corrected). $\boldsymbol{b}$, The conjunction map shows a region of left dIPFC that correlates with $\mathrm{HR}$ and had increased coupling with vmPFC at the time of decision. Voxels in red are correlated with the parametric regressor for HR at $p<0.005$, uncorrected, and 20 voxel extent. Voxels in yellow show increased coupling with vmPFC (PPI) at the time of decision $(p<$ 0.005 , uncorrected; 20 voxel extent). The conjunction of these two contrasts is shown in green

abilities can be used to define a multinomial distribution over model space from which the likelihood that a specific model generated the data of a randomly selected subject can be estimated. This procedure permits the computation of the exceedance probability (EP) for each model in the comparison set. Note that EPs represent the posterior belief that a model is more likely to be correct than all others in the comparison set, that the posterior probability of one model versus another is given by the ratio of their EPs, and that EPs sum to one over all models in the comparison set, which implies that EPs tend to decrease with the number of models tested.

Fourth, we constructed and compared eight additional DCM models based on the fixed connectivity of the most likely model from the first BMS procedure. The set of eight models tested was chosen to cover a wide range of potential interregional interactions. These models varied in terms of whether or not activity in a given node could modulate the coupling between two other nodes. The models tested are diagrammed in Figure 7. We used the same BMS method described above to identify the most likely model from this set of eight (Fig. 6).

Note that we performed the Bayesian model selection process in two steps (optimize among 30 fixed connection models, then compare eight nonlinear models) instead of the usual single step, in order to reduce the number of models that had to be compared with a tractable number. Our solution provides an approximation for the optimal model that would have been selected from the full one-step search that includes all of the combinations of the classes of models.

Fifth, we used Bayesian parameter averaging (Lee, 1989; Kasess et al., 2010) to estimate the magnitudes and probabilities of each coupling parameter, as well as the magnitudes and effects with which the connections are modulated by different task events or activity in other regions. Briefly, this method uses the estimated posterior densities for each connectivity parameter for each subject, and combines them to obtain a single conditional density for the group by treating the posterior density 
of the first subject as the prior density for the second subject and continuing this process up to the $n$th subject. Note that this procedure is commutative and does not depend on the order in which subjects' data is entered. Furthermore, under the maintained hypothesis that the underlying densities being estimated are Gaussian, it is possible to simplify this procedure and weight the subject-specific conditional parameter densities by their precision and then sum them across subjects rather than estimating the posterior density of each subject using a prior based on the densities of the preceding subjects. The incorporation of the within-subjects estimation precision is an advantage of Bayesian parameter averaging over frequentist approaches (e.g., one-sample $t$ test) that do not include any measure of withinsubject variance.

Sixth, to carry out the simulations of vmPFC responses described in Figure $6 e$, we solved the system of differential equations describing the neuronal activity implied by the estimated DCM, as well as that of several variations of the model described in the Results, below. In particular, we simulated the response of the four nodes in the network to a single trial of $1 \mathrm{~s}$ duration, to a food with a taste rating of 0.5 , and health ratings were drawn from the set $[-2,-1,1,2]$. The taste level was chosen to represent a food with relatively neutral taste while still providing a nonzero driving input to vmPFC. The measure of vmPFC activity reported in Figure $6 e$ is computed as the (signed) area under the response curve.

\section{Results}

\section{Behavioral results}

We analyzed the impact of attention cue on dietary choices in two different, but complementary, ways.

First, we estimated a mixed-effects linear regression model of stimulus values on $\mathrm{HR}$, $\mathrm{TR}$, a dummy for $\mathrm{HC}$, a regressor for $\mathrm{HC}$ interacted with $\mathrm{HR}\left(\mathrm{HR}^{\star} \mathrm{HC}\right)$, a regressor for $\mathrm{HC}$ interacted with $\mathrm{TR}\left(\mathrm{TR}^{\star} \mathrm{HC}\right)$, a dummy for TC, a regressor for TC interacted with $\mathrm{HR}\left(\mathrm{HR}^{\star} \mathrm{TC}\right)$, and a regressor for TC interacted with TR (TR*TC). The stimulus values were measured with the fourpoint behavioral response entered at the time of decision (Strong No $=-2$ to Strong Yes $=2$ ). Note that in this model, the NC serves as the baseline, and the $\mathrm{HC}$ and TC regressors measure deviations from NC. Figure $2 a$ summarizes the results. There were main effects of $\mathrm{HR}\left(t_{32}=3.98\right.$; $p<$ $0.001)$, TR $\left(t_{32}=13.99 ; p<0.001\right)$, and HC $\left(t_{32}=-3.17 ; p<0.005\right)$. The interactions of $\mathrm{HR}^{\star} \mathrm{HC}\left(t_{32}=4.90 ; p<0.001\right), \mathrm{HR}^{\star} \mathrm{TC}$ $\left(t_{32}=-2.28 ; p<0.05\right)$, and $\mathrm{TR}^{\star} \mathrm{HC}\left(t_{32}=\right.$ $-3.05 ; p<0.005)$ were also significant. The data show that during $\mathrm{HC}$, subjects were more responsive to the health features of the stimuli than in $\mathrm{NC}$, and less responsive to the taste features. During TC they were less
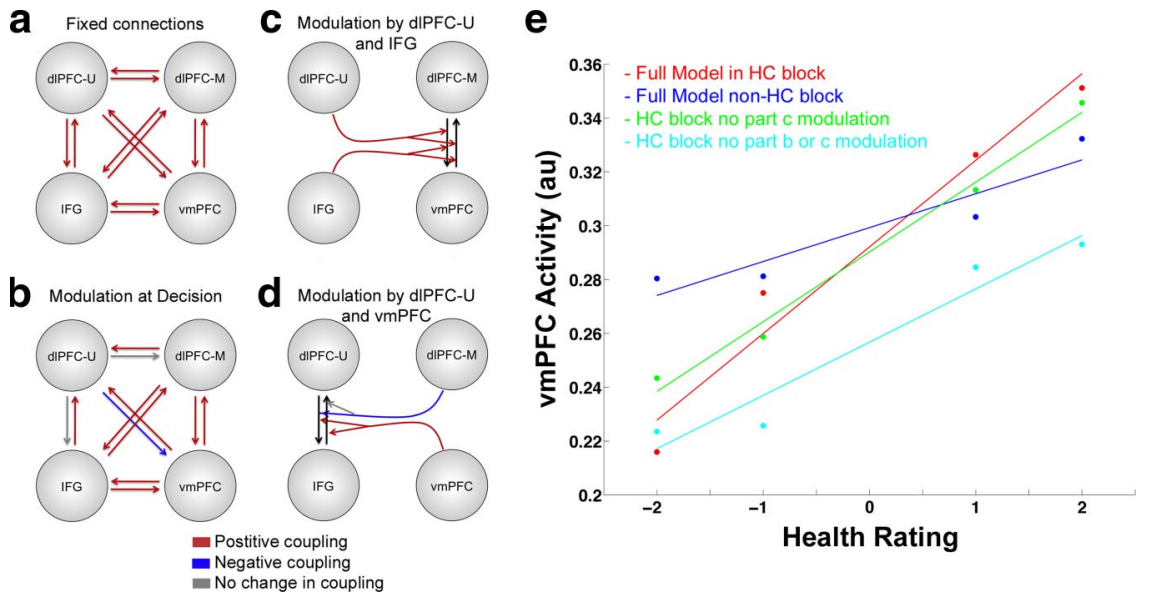

Figure 6. Dynamic causal modeling of interregional coupling. a, Fixed coupling between dIPFC-U (BA 8/9), IFG (BA 46/47), dIPFC-M (BA 9), and vmPFC. $\boldsymbol{b}$, Coupling modulation at decision time in all blocks. c, Modulation of the coupling between dIPFC-M and vmPFC by dIPFC-U and IFG. $\boldsymbol{d}$, Modulation of the coupling between dIPFC- $U$ and IFG by dIPFC-M and vmPFC. Red arrows indicate positive coupling or modulation with $\geq 90 \%$ probability. Blue arrows indicate negative modulation with $\geq 90 \%$ probability. Gray arrows indicate pathways were the probability of modulation is $<90 \%$. $\boldsymbol{e}$, Simulations of vmPFC responses at the time of decision to foods of different healthiness under the assumptions of the parameters of the estimated DCM. The taste of the food items is kept constant in these simulations. The red dots and line represent vmPFC activity simulated using the full model in an $\mathrm{HC}$ block. Blue represents vmPFC activity from the full model in a non-HC block. Green and cyan lines show simulated vmPFC activity in $\mathrm{HC}$ blocks when specific aspects of the DCM model have been removed. Green shows simulated activity when the modulation parameters of the connections between vmPFC and dIPFC-M by dIPFC- $U$ and IFG $(\boldsymbol{c})$ are set to zero. The cyan line shows simulated activity when the modulation parameters at the time of choice $(\boldsymbol{b})$ and the modulation in care set to zero. The $y$-axis shows the vmPFC response in arbitrary units. All lines represent the least-squares linear fit to the simulated vmPFC signal. a

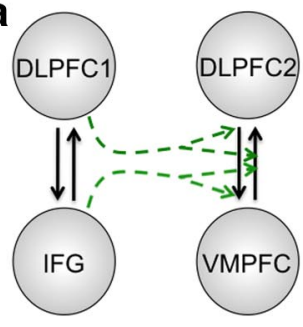

d

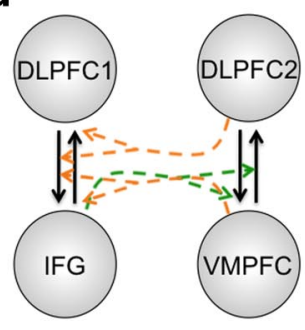

g

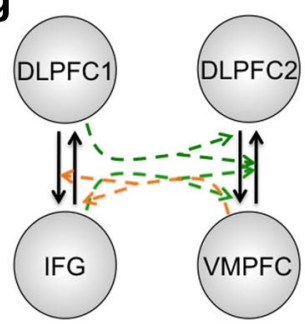

b

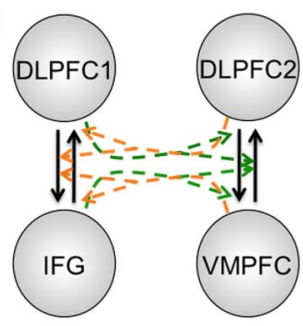

e

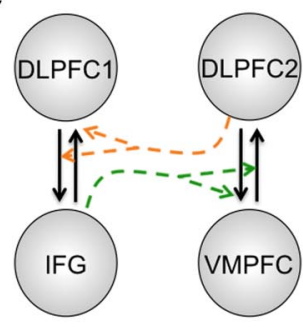

h

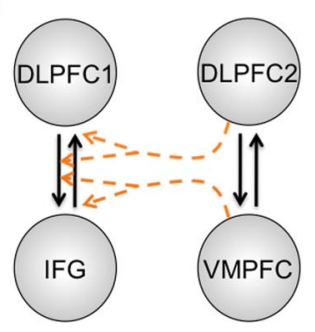

C

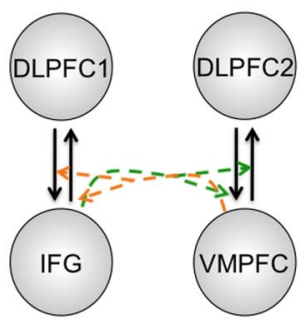

$\mathbf{f}$

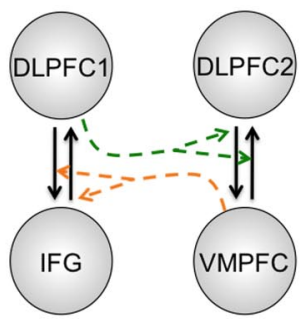

i

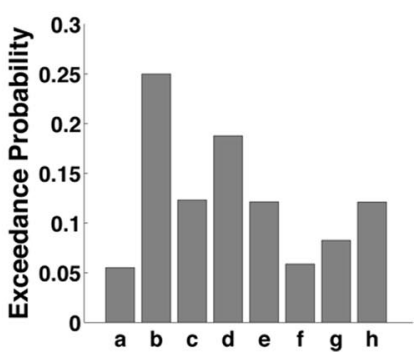

Figure 7. DCM models. $\boldsymbol{a}-\boldsymbol{h}$, These diagrams show eight alternative models of coupling modulation by different regions. The driving inputs and fixed connections for all models are described in the main text. Dotted arrows represent potential modulation of the coupling between two regions by a third region. Colors are used only to indicate separate modulation pathways. $\boldsymbol{i}$, Bar graph showing the exceedance probability for each model on the $y$-axis. The labels on the $x$-axis correspond to the letters assigned to each diagram. 
sensitive to the health features than in NC, but they were not significantly more sensitive to the taste features.

Second, we classified foods into four pairings based on the subjects' attribute ratings: unhealthy-untasty, healthy-untasty, unhealthy-tasty, and healthy-tasty (Fig. $2 b$ ). There was no significant difference in choices over healthy-tasty foods between any of the conditions. In HC blocks, subjects were less likely to eat unhealthy-tasty $\left(t_{32}=-3.55 ; p<0.005\right)$ and unhealthy-untasty foods $\left(t_{32}=-3.23 ; p<0.005\right)$, but more likely to eat healthyuntasty foods $\left(t_{32}=2.65 ; p<0.05\right)$. There were no significant differences between NC and TC. Overall, subjects were less likely to eat foods during $\mathrm{HC}$ blocks ( $34 \%$ yes) relative to $\mathrm{NC}$ blocks ( $50 \%$ yes; paired $t$ test: $\left.t_{32}=-5.56, p<0.001\right)$. Note that the lack of a difference in choices between TC and NC blocks is not inconsistent with the results in Figure $2 a$ because the two analyses are based on different response variables: the first set of results is about changes in stimulus values, whereas the second is about Yes/No choices.

There were no significant differences in reaction times between conditions (mean $r t=1526 \mathrm{~ms}, \mathrm{SD}=289 \mathrm{~ms}$ ). Because we found effects for HC, but not TC, blocks on behavioral choices, the fMRI analyses below focus on the effects of the health cues.

\section{Neuroimaging results}

Correlates of stimulus values

The first step in the fMRI analysis identified regions that correlated with the stimulus values assigned to foods at the time of choice, independent of attention condition. To do this, we estimated a GLM of BOLD activity in which responses during the decision period were modulated by stimulus values and which assumed that the neural responses were common across the three attention conditions. We found that activity in vmPFC and posterior cingulate cortex (PCC) correlated positively with stimulus values ( $p<0.05$ corrected) (Fig. $3 a$, Table 1$)$.

We performed an additional post hoc analysis to further investigate the nature of the stimulus value signals in these two regions. Activity in regions representing the overall stimulus value should not change as a function of the attention condition. As a result, the correlation between activity in this area and the reported stimulus value should not be different across conditions. We tested this hypothesis by estimating another model (GLM 3), which allowed the BOLD response to differ by condition. We then computed the average beta values for the stimulus value modulator in each condition by averaging within a $4 \mathrm{~mm}$ sphere centered on the subject's individual peak within the group peak for the contrast of all decisions modulated by stimulus value in GLM 1. The tests and post hoc tests showed no significant differences between the three conditions in the strength of the correlations between stimulus values and vmPFC activity $\left[\max t_{32}\right.$ value $=1.71$, not significant (n.s.)] or PCC activity $\left(\max t_{32}\right.$ value $=-0.72$, n.s.) (Fig. $\left.3 b\right)$.

Lastly, the bar graphs in Figure $3 c$ display the amount of activity in vmPFC and PCC at each level of stimulus value for visualization purposes only and do not represent a statistical analysis.

\section{Correlates of health and taste ratings}

Next, we identified areas that correlated with subjects' health and taste ratings at the time of decision during NC blocks only. This was done by estimating a second GLM that parallels the behavioral model described above. Using one-sample $t$ tests against zero at the group level, we found that regions of vmPFC and dlPFC (BA 8) correlated with health ratings, while regions of vmPFC and PCC correlated with taste ratings $(p<0.05$, corrected) (Fig. 4a,b; Tables 2, 3). A conjunction analysis showed a significant overlap in the extent of the signals for stimulus value, health, and taste ratings in vmPFC, but not in PCC, at our omnibus threshold of $p<0.05$, corrected (Fig. $4 a$ ). The results of this conjunction, together with the data shown in Figure 3, suggest that the activity in vmPFC resembles the encoding of a stimulus value signal, but that the activity in PCC does not. As a result, the subsequent analyses focus on the properties of the value signals in vmPFC.

\section{Correlation between the responsiveness of $v m P F C$ activity and} behavior to health cues

The third step in the imaging analysis investigated whether the impact of health cues on behavior correlated with the extent to which attention to health increased the weighting of healthiness in vmPFC value signals as measured by the $\mathrm{HR}^{\star} \mathrm{HC}$ coefficients in GLM 2. To do this, we estimated a robust linear regression of each individual's neural measure on the individual's behavioral measure and found a significant relationship between the behavioral and neural $\mathrm{HR}^{\star} \mathrm{HC}$ coefficients (robust regression coefficient $=0.69, p<0.005$ ) (Fig. $4 c$ ).

We also performed an exploratory analysis examining the relationship between the behavioral and vmPFC neural coefficients for all ratings and interactions, but no others were significant after applying Bonferroni corrections.

\section{Potential effectors of the attention-induced changes}

The fourth step in the fMRI analysis identified regions that are candidates for playing a role in implementing the computational changes associated with attention cues. We did this in two parts.

First, we looked for regions that exhibited an increase in activity in $\mathrm{HC}$ or TC blocks compared with the NC control blocks. Such regions could potentially affect choice by modulating valuation processes in other areas. We found stronger activity during HC compared with NC in left IFG (BA 47/46), a more posterior region of left dlPFC (BA 8/9), bilateral parietal cortex, and right cerebellum ( $p<0.05$, corrected) (Fig. $5 a$, Table 4). A comparison of TC minus NC found more activity in bilateral parietal cortex and the left inferior temporal gyrus ( $p<0.05$, corrected) (Table 5).

Second, we looked for areas that both correlated with the health ratings and exhibited increased functional connectivity with vmPFC. Regions satisfying both criteria are potential candidates for passing health information to vmPFC that can be integrated into the stimulus value signals. We began this part of the analysis by conducting a PPI analysis to identify regions that exhibited increased connectivity with the vmPFC ROI that correlated with stimulus value (Table 6). Next, we performed a conjunction analysis to identify regions that correlated with HRs and had increased coupling with vmPFC at the time of decision. Only one region in left dlPFC (BA9) showed both effects (conjunction threshold, $p<0.005$, uncorrected; 20 voxel extent) (Fig. 5b). Note that neither of the individual contrasts used in this conjunction showed any overlap with the area of BA 8/9 where activity increased during health trials, even at a liberal threshold of $p<$ 0.01 , uncorrected, suggesting that the two subregions are involved in distinct computations.

These results suggest that three distinct areas of left lateral PFC exhibit activity patterns consistent with a role in modulating the changes in the valuation circuitry triggered by attention cues: regions IFG (BA 47/46) and dlPFC (BA 8/9), that were more active during the health condition, and a distinct area of dlPFC (BA 9) that correlated with HRs and showed increased coupling with vmPFC at the time of choice. To simplify the Discussion 
Table 7. Estimated DCM coupling parameters

\begin{tabular}{|c|c|c|c|c|}
\hline & dIPFC-U $\Rightarrow$ & $\mathrm{IFG} \Rightarrow$ & $\mathrm{dIPFC}-\mathrm{M} \Rightarrow$ & $\mathrm{vmPFC} \Rightarrow$ \\
\hline \multicolumn{5}{|c|}{ Fixed coupling } \\
\hline dIPFC-U & -1 & $0.25\left(\sigma^{2}=0.00022 ; P_{c}=1\right)$ & $0.45\left(\sigma^{2}=0.00025 ; P_{c}=1\right)$ & $0.29\left(\sigma^{2}=0.0003 ; P_{c}=1\right)$ \\
\hline $\mathrm{IFG}$ & $0.13\left(\sigma^{2}=0.00022 ; P_{c}=1\right)$ & -1 & $0.3\left(\sigma^{2}=0.00024 ; P_{c}=1\right)$ & $0.36\left(\sigma^{2}=0.0003 ; P_{c}=1\right)$ \\
\hline dIPFC-M & $0.22\left(\sigma^{2}=0.00024 ; P_{c}=1\right)$ & $0.21\left(\sigma^{2}=0.00023 ; P_{c}=1\right)$ & -1 & $0.31\left(\sigma^{2}=0.00031 ; P_{c}=1\right)$ \\
\hline vmPFC & $0.16\left(\sigma^{2}=0.00022 ; P_{c}=1\right)$ & $0.31\left(\sigma^{2}=0.00022 ; P_{c}=1\right)$ & $0.23\left(\sigma^{2}=0.00025 ; P_{c}=1\right)$ & -1 \\
\hline \multicolumn{5}{|c|}{ Modulation by decision } \\
\hline dIPFC-U & $0.3\left(\sigma^{2}=0.00034 ; P_{\mathrm{m}}=1\right)$ & $0.07\left(\sigma^{2}=0.00032 ; \mathrm{P}_{\mathrm{m}}=1\right)$ & $0.14\left(\sigma^{2}=0.00037 ; P_{\mathrm{m}}=1\right)$ & $0.04\left(\sigma^{2}=0.00038 ; P_{\mathrm{m}}=0.99\right)$ \\
\hline IFG & $-0.02\left(\sigma^{2}=0.00033 ; P_{\mathrm{m}}=0.81\right)$ & $0.58\left(\sigma^{2}=0.00031 ; P_{\mathrm{m}}=1\right)$ & $0.14\left(\sigma^{2}=0.00035 ; P_{\mathrm{m}}=1\right)$ & $0.07\left(\sigma^{2}=0.00039 ; P_{\mathrm{m}}=1\right)$ \\
\hline dIPFC-M & $0.01\left(\sigma^{2}=0.00032 ; P_{\mathrm{m}}=0.61\right)$ & $0.09\left(\sigma^{2}=0.00031 ; P_{\mathrm{m}}=1\right)$ & $0.46\left(\sigma^{2}=0.00035 ; P_{\mathrm{m}}=1\right)$ & $0.06\left(\sigma^{2}=0.00038 ; P_{\mathrm{m}}=1\right)$ \\
\hline vmPFC & $-0.04\left(\sigma^{2}=0.00032 ; P_{\mathrm{m}}=0.98\right)$ & $0.08\left(\sigma^{2}=0.00031 ; P_{\mathrm{m}}=1\right)$ & $0.04\left(\sigma^{2}=0.00036 ; P_{\mathrm{m}}=0.98\right)$ & $0.19\left(\sigma^{2}=0.00039 ; P_{\mathrm{m}}=1\right)$ \\
\hline \multicolumn{5}{|c|}{ Modulation by vmPFC } \\
\hline dIPFC-U & - & $1.04\left(\sigma^{2}=0.00001 ; P_{\mathrm{m}}=1\right)$ & - & - \\
\hline IFG & $0.83\left(\sigma^{2}=0.01 ; P_{\mathrm{m}}=1\right)$ & - & - & - \\
\hline \multicolumn{5}{|c|}{ Modulation by dIPFC-M } \\
\hline dIPFC-U & - & $-0.07\left(\sigma^{2}=0.01 ; P_{\mathrm{m}}=0.81\right)$ & - & - \\
\hline IFG & $-0.47\left(\sigma^{2}=0.01 ; P_{\mathrm{m}}=1\right)$ & - & - & - \\
\hline dIPFC-M & - & - & - & - \\
\hline vmPFC & - & - & - & - \\
\hline \multicolumn{5}{|c|}{ Modulation by dIPFC-U } \\
\hline dIPFC-U & - & - & - & - \\
\hline IFG & - & - & - & - \\
\hline dIPFC-M & - & - & - & $0.9\left(\sigma^{2}=0.01 ; P_{\mathrm{m}}=1\right)$ \\
\hline vmPFC & - & - & $0.41\left(\sigma^{2}=0.01 ; P_{\mathrm{m}}=1\right)$ & - \\
\hline \multicolumn{5}{|c|}{ Modulation by IFG } \\
\hline dIPFC-U & - & - & - & - \\
\hline IFG & - & - & - & - \\
\hline dIPFC-M & - & - & - & $1.54\left(\sigma^{2}=0.01 ; P_{\mathrm{m}}=1\right)$ \\
\hline vmPFC & - & - & $1.22\left(\sigma^{2}=0.01 ; P_{\mathrm{m}}=1\right)$ & - \\
\hline
\end{tabular}

Coupling flows from the region listed at the top of each column to those listed in each row.

$\sigma^{2}$, Sample variance; $P_{c}$, probability that the absolute value of the coupling parameter is greater than zero; $P_{\mathrm{m}}$, probability that the coupling parameter is modulated by task condition. Probabilities are rounded to two decimal places and those parameters whose probability is greater than $90 \%$ are shown in bold.

below, we refer to the first dlPFC (BA 8/9) area as dlPFC-U and to the second area of dlPFC (BA 9) as dlPFC-M.

Functional interactions between lateral PFC and $v m P F C$

The final step in the fMRI analysis examined the mechanisms through which the coordinated activity in these three areas might modulate the value signals in vmPFC. We did this by estimating nonlinear DCM of effective connectivity between the areas of lateral PFC specified above and the vmPFC. This was done in several steps.

First, we specified 30 separate models of fixed connectivity between vmPFC and the three areas of lateral PFC identified above. These models included the fully connected model, as well as all other possible connectivity patterns created by removing one to four bidirectional connections between the four nodes of the full model. In addition to the fixed connections, the model also allowed for the strength of each connection to change independently at the time of decision. All of the models assumed the following exogenous driving inputs of activity based on the GLM results above: (1) an input to vmPFC at choice proportional to TRs, (2) an input to dlPFC-M at choice proportional to HRs, (3) an input to dlPFC-U and IFG of constant unit magnitude during the choice phase, and (4) an input of constant value to dlPFC-U and IFG that was active during the entire length of the health block. We used Bayesian model selection to identify the most probably model in this set. The fully connected model was the most probable (Fig. $6 a$ ), with an EP of 0.52 relative to the other 29 models (which ranged in EP from 0.0001 to 0.26 ).

Second, we performed a further refinement of the DCM by allowing nodes to modulate the coupling between two other nodes. We specified eight alternative models with this type of modulation, which covered a wide range of potential interregional interactions (Fig. 7). Note that these modulation parameters capture the degree to which changes in the activity of one region modulate the coupling between two other regions, and that they are not tied to particular events within a trial. However, these modulation parameters can play an important role in changing value signals if activity in their source regions varies at different points in the task. We again used Bayesian model selection to identify the model shown in Figure 6 as the most likely. This model had an EP of 0.25 relative to the other seven models, whose values ranged from 0.06 to 0.19 .

Third, we used Bayesian parameter averaging to estimate the posterior probabilities of each parameter of the most likely DCM. The results are described in Table 7. The fixed bilateral connections between all regions were positive and significant (Fig. $6 a$, Table 7), indicating that at baseline there was positive coupling between all four regions. At the time of decision (regardless of attention condition), the bidirectional coupling between the IFG, dlPFC-M, and vmPFC regions increased, indicating that activity in these regions is more tightly coupled when making a choice (Fig. 6b, Table 7). In contrast, the coupling from dlPFC-U to vmPFC was negatively modulated at the time of decision, indicating that activity in vmPFC is less responsive to signals from dlPFC-U during choice. However, the coupling from vmPFC to dlPFC-U increased during decisions, suggesting that the activity in dlPFC-U is more responsive to value signals reflected in vmPFC activity at the time of choice. In addition, the bidirectional coupling between DLPFC-U and IFG was positively modulated by vmPFC activity (Fig. $6 d$, Table 7), again consistent with the idea that activity in these two regions is responsive to value 
signals reflected in vmPFC activity. Moreover, the coupling from dIPFC-U to IFG was negatively modulated by activity in the dIPFC-M region that reflected health ratings, suggesting that the signaling from dIPFC-U to IFG might be proportional to the amount of modulation required (Table 7). Higher dlPFC-M activity would indicate a healthier food item and less need to reduce the stimulus value to refrain from eating the food. Finally, activity in both dlPFC-U and IFG positively modulated the bidirectional coupling between dlPFC-M and vmPFC (Fig. 6c, Table 7). This is consistent with the idea that dIPFC-U and IFG modulate vmPFC activity by changing the strength of vmPFC interactions with regions encoding various stimulus attributes (e.g., health or taste).

Fourth, we conducted simulations using the estimated DCM parameters of the best model to visualize the connectivity results and their implications for how regions of lateral PFC might modulate stimulus value computations in vmPFC, resulting in healthier choices. The first step (Fig. 6e, red) involved solving the system of differential equations underlying the fitted DCM to characterize vmPFC responses to foods in the HC blocks as function of different health ratings (while holding the taste value constant). We then performed the same exercise to simulate predicted responses in non-health consideration trials (Fig. 6e, blue). Consistent with the behavioral results, the selected DCM shows that vmPFC responses are more sensitive to health ratings in HC blocks compared with non-health blocks. To investigate the role of modulations in coupling at decision time or via different regions, we performed two additional simulations. First, we simulated vmPFC responses during $\mathrm{HC}$ blocks in the absence of any modulation by dIPFC-U or IFG on the coupling between dIPFC-M and vmPFC. Removing these modulation parameters from the model prevents activity in dIPFC-U and IFG from influencing the degree to which health-rating signals from dlPFC-M are conveyed to vmPFC (Fig. 6e, green). Second, we removed the modulation of all connections at the time of choice. The removal of both forms of modulation decreased the responsiveness of vmPFC to the health ratings (Fig. 6e, cyan). This shows that, under the assumptions of the most likely DCM, the modulation of functional coupling plays a critical role in shifting the stimulus value signals in vmPFC to become more sensitive to the healthiness of foods.

\section{Discussion}

This study builds on previous work by our group on the computational and neurobiological basis of self-control (Hare et al., 2009). As mentioned in the Introduction, we found several important differences in the patterns of activity in vmPFC and dIPFC between subjects exhibiting high and low levels of dietary self-control. In the current study, we addressed two related open questions: can exogenous cues that draw attention to the health features of foods increase healthy eating and do such manipulations of attention operate by changing the extent to which the dIPFC modulates vmPFC activity so that the value signals computed there reflect the healthiness of foods?

Our behavioral and neural evidence is largely consistent with both hypotheses. Behaviorally, we found that cues that direct attention to the health features of foods at the time of choice increased the extent to which health was taken into account by non-dieting individuals. We found no differences in choices on trials where the cues focused attention on the tastiness of foods. One likely explanation for this asymmetry is that subjects in this study reported not adhering to any dietary restrictions and already highly weighted the taste features of the foods in the natural condition. Thus, attracting further attention to taste might not increase its impact on the stimulus value signals or choices. In contrast, and consistent with Figure $2 a$, the health features are likely to be underweighted in the natural condition, meaning that there is room for the health attention cues to increase health weighting in the computation of stimulus values.

In addition to changing behavior, health cues also influenced vmPFC activity during choice. Consistent with many previous studies, activity in a region of vmPFC encompassing the medial orbitofrontal and ventral anterior cingulate cortex correlated with value computations at the time of choice in all conditions (Padoa-Schioppa and Assad, 2006; Kable and Glimcher, 2007; Plassmann et al., 2007, 2010; Tom et al., 2007; Hare et al., 2008, 2010; Boorman et al., 2009; Chib et al., 2009; Kennerley et al., 2009; Levy et al., 2010; Litt et al., 2011). Furthermore, consistent with the hypothesis that vmPFC signals integrate the values of stimulus features (Basten et al., 2010; Kahnt et al., 2010), this area was responsive to both taste and health ratings during natural trials. More importantly, increases in healthy choices during HC blocks were correlated across subjects with increases in the degree to which health ratings were reflected in vmPFC activity. A similar relationship between the weight given to health ratings in vmPFC and the impact of health ratings on decisions was seen previously in dieters (Hare et al., 2009). This result is consistent with the idea that a natural way to exercise self-control is to modulate value signals so that they properly weight long-term considerations such as health. Our results suggest that health attention cues induce improved dietary choices by increasing the weight that healthiness receives in the vmPFC value signals.

The presence of health attention cues was also associated with increased activity in two regions of left lateral prefrontal cortex [dlPFC-U (BA 8/9) and IFG (BA 47/46)] that are anatomically proximate to those previously implicated in self-control in actively dieting individuals (Hare et al., 2009) and have been shown to play a role in cognitive control, working memory, and emotion regulation (Duncan and Owen, 2000; Miller and Cohen, 2001; Ochsner and Gross, 2005; Badre and Wagner, 2007). Dynamic causal modeling analyses of functional coupling showed that dIPFC-U and IFG (areas that were more active during the health cues but did not correlate with HRs) modulated the coupling between dlPFC-M (where activity correlated with HRs) and vmPFC. Furthermore, the coupling between dIPFC-U and IFG regions was modulated by the level of activity in the vMPFC and dIPFC-M. This is consistent with the idea that healthier foods might require less modulation and therefore less interaction between left dIPFC and IFG. The changes in coupling between lateral PFC and vmPFC that are associated with healthier choices in non-dieting individuals in the current study also parallel the findings for active dieters in our previous study (Hare et al., 2009). The similarity between the neurophysiological processes induced by health attention cues and endogenous self-control suggests that health cues may influence behavior by exogenously activating the same networks that dieters activate on their own when successfully using self-control.

The fact that exogenous cues designed to modify the relative amount of attention paid to various stimulus features can affect choices is consistent with a growing body of experimental and theoretical work in psychology and neuroscience that suggests values are computed dynamically at the time of choice and attention can affect the value-construction process (Busemeyer and Towsend, 1993; Ratcliff and Rouder, 2000; Roe et al., 2001; Usher and McClelland, 2001; Ratcliff et al., 2003; Shimojo et al., 2003; Rieskamp et al., 2006; Bogacz, 2007; Armel and Rangel, 2008; Armel et al., 2008; Ratcliff and McKoon, 2008; Krajbich et al., 
2010). The basic idea in these models is that the decision-making circuitry assigns values to the stimuli under consideration at every point in time until a choice is made. An implicit assumption in these models is that the values at each point in time are a weighted sum of the stimulus attributes, with the weights depending on how attention is allocated among the features. Our findings provide empirical evidence that directing attention to a specific feature can increase its relative weighting during value computation.

A small but potentially useful methodological innovation of this study was to use simulation methods to understand the role that the modulation of various connections played in changing the pattern of activation in vmPFC during the health cue trials. The simulations are useful because minimally realistic connectivity models, such as those posited by DCM, involve nonlinear modulation and bidirectional task-dependent feedback, leading to complex interaction dynamics across regions that are often hard to understand. The simulation method allowed us to compare the predictions for vmPFC activity from the best-fitting model when specific components of the model are turned off. These simulations showed that the modulation of various connections to vmPFC can change the relative weightings of health and taste factors in the vmPFC signal that is believed to drive choice. Of course, these predicted effects can only be as accurate as the models we use to simulate them. Nevertheless, we hope that this type of simulation exercise will prove increasingly useful in fMRI as the complexity and realism of the networks studied increases.

Our findings have potentially useful implications for future therapeutic and public policy interventions. The results suggest that methods that help the individual to direct attention toward the long-term features of the stimuli (e.g., health in the case of food) may lead to better decisions in situations where immediate gratification is at odds with long-term well being. These findings also suggest a possible explanation, although a purely speculative one at this point, for why graphical cigarette warnings are associated with higher indicators of smoking cessation and prevention than less salient text warnings that convey similar information but may be less effective in capturing attention and, therefore, have less impact on value computations (White et al., 2008; Borland et al., 2009).

\section{References}

Armel KC, Rangel A (2008) The impact of computation time and experience on decision values. Am Econ Rev 98:163-168.

Armel KC, Beaumel A, Rangel A (2008) Biasing simple choices by manipulating relative visual attention. Judgment Decision Making 3:396-403.

Badre D, Wagner AD (2007) Left ventrolateral prefrontal cortex and the cognitive control of memory. Neuropsychologia 45:2883-2901.

Basten U, Biele G, Heekeren HR, Fiebach CJ (2010) How the brain integrates costs and benefits during decision making. Proc Natl Acad Sci U S A 107:21767-21772.

Bettman JR, Luce MF, Payne JW (1998) Constructive consumer choice processes. J Consum Res 25:187-217.

Bogacz R (2007) Optimal decision-making theories: linking neurobiology with behaviour. Trends Cogn Sci 11:118-125.

Boorman ED, Behrens TE, Woolrich MW, Rushworth MF (2009) How green is the grass on the other side? Frontopolar cortex and the evidence in favor of alternative courses of action. Neuron 62:733-743.

Borland R, Wilson N, Fong GT, Hammond D, Cummings KM, Yong HH, Hosking W, Hastings G, Thrasher J, McNeill A (2009) Impact of graphic and text warnings on cigarette packs: findings from four countries over five years. Tob Control 18:358-364.

Brainard DH (1997) The Psychophysics Toolbox. Spat Vis 10:433-436.

Busemeyer JR, Towsend JT (1993) Decision field theory: a dynamic- cognitive approach to decision making in an uncertain environment. Psychol Rev 100:432-459.

Chib VS, Rangel A, Shimojo S, O’Doherty JP (2009) Evidence for a common representation of decision values for dissimilar goods in human ventromedial prefrontal cortex. J Neurosci 29:12315-12320.

Deichmann R, Gottfried JA, Hutton C, Turner R (2003) Optimized EPI for fMRI studies of the orbitofrontal cortex. Neuroimage 19:430-441.

Duncan J, Owen AM (2000) Common regions of the human frontal lobe recruited by diverse cognitive demands. Trends Neurosci 23:475-483.

Gitelman DR, Penny WD, Ashburner J, Friston KJ (2003) Modeling regional and psychophysiologic interactions in fMRI: the importance of hemodynamic deconvolution. Neuroimage 19:200-207.

Hare TA, O'Doherty J, Camerer CF, Schultz W, Rangel A (2008) Dissociating the role of the orbitofrontal cortex and the striatum in the computation of goal values and prediction errors. J Neurosci 28:5623-5630.

Hare TA, Camerer CF, Rangel A (2009) Self-control in decision-making involves modulation of the vmPFC valuation system. Science 324: 646-648.

Hare TA, Camerer CF, Knoepfle DT, O’Doherty JP, Rangel A (2010) Value computations in ventral medial prefrontal cortex during charitable decision making incorporate input from regions involved in social cognition. J Neurosci 30:583-590.

Kable JW, Glimcher PW (2007) The neural correlates of subjective value during intertemporal choice. Nat Neurosci 10:1625-1633.

Kable JW, Glimcher PW (2009) The neurobiology of decision: consensus and controversy. Neuron 63:733-745.

Kahnt T, Heinzle J, Park SQ, Haynes JD (2010) Decoding different roles for vmPFC and dlPFC in multi-attribute decision making. Neuroimage 56:709-715.

Kasess CH, Stephan KE, Weissenbacher A, Pezawas L, Moser E, Windischberger C (2010) Multi-subject analyses with dynamic causal modeling. Neuroimage 49:3065-3074.

Kennerley SW, Dahmubed AF, Lara AH, Wallis JD (2009) Neurons in the frontal lobe encode the value of multiple decision variables. J Cogn Neurosci 21:1162-1178.

Krajbich I, Armel C, Rangel A (2010) Visual fixations and the computation and comparison of value in goal-directed choice. Nat Neurosci 13:1292-1298.

Lee PM (1989) Bayesian statistics. New York: Oxford UP.

Levy I, Snell J, Nelson AJ, Rustichini A, Glimcher PW (2010) The neural representation of subjective value under risk and ambiguity. J Neurophysiol 103:1036-1047.

Liberman N, Trope Y (2008) The psychology of transcending the here and now. Science 322:1201-1205.

Litt A, Plassmann H, Shiv B, Rangel A (2011) Dissociating valuation and saliency signals during decision-making. Cereb Cortex 21:95-102.

Miller EK, Cohen JD (2001) An integrative theory of prefrontal cortex function. Annu Rev Neurosci 24:167-202.

Montague PR, Berns GS (2002) Neural economics and the biological substrates of valuation. Neuron 36:265-284.

Ochsner KN, Gross JJ (2005) The cognitive control of emotion. Trends Cogn Sci 9:242-249.

Padoa-Schioppa C, Assad JA (2006) Neurons in the orbitofrontal cortex encode economic value. Nature 441:223-226.

Plassmann H, O’Doherty J, Rangel A (2007) Orbitofrontal cortex encodes willingness to pay in everyday economic transactions. J Neurosci 27:9984-9988.

Plassmann H, O'Doherty JP, Rangel A (2010) Appetitive and aversive goal values are encoded in the medial orbitofrontal cortex at the time of decision making. J Neurosci 30:10799-10808.

Rangel A, Hare T (2010) Neural computations associated with goal-directed choice. Curr Opin Neurobiol 20:262-270.

Rangel A, Camerer C, Montague PR (2008) A framework for studying the neurobiology of value-based decision making. Nat Rev Neurosci 9:545-556.

Ratcliff R, McKoon G (2008) The diffusion decision model: theory and data for two-choice decision tasks. Neural Comput 20:873-922.

Ratcliff R, Rouder JN (2000) A diffusion model account of masking in two-choice letter identification. J Exp Psychol Hum Percept Perform 26:127-140.

Ratcliff R, Cherian A, Segraves M (2003) A comparison of macaque behav- 
ior and superior colliculus neuronal activity to predictions from models of two-choice decisions. J Neurophysiol 90:1392-1407.

Rieskamp J, Busemeyer JR, Mellers BA (2006) Extending the bounds of rationality: evidence and theories of preferential choice. J Econ Lit 44:631-661.

Roe RM, Busemeyer JR, Townsend JT (2001) Multialternative decision field theory: a dynamic connectionist model of decision making. Psychol Rev 108:370-392.

Rushworth MF, Mars RB, Summerfield C (2009) General mechanisms for making decisions? Curr Opin Neurobiol 19:75-83.

Schoenbaum G, Roesch MR, Stalnaker TA, Takahashi YK (2009) A new perspective on the role of the orbitofrontal cortex in adaptive behaviour. Nat Rev Neurosci 10:885-892.

Shimojo S, Simion C, Shimojo E, Scheier C (2003) Gaze bias both reflects and influences preference. Nat Neurosci 6:1317-1322.
Stephan KE, Kasper L, Harrison LM, Daunizeau J, den Ouden HE, Breakspear M, Friston KJ (2008) Nonlinear dynamic causal models for fMRI. Neuroimage 42:649-662.

Stephan KE, Penny WD, Daunizeau J, Moran RJ, Friston KJ (2009) Bayesian model selection for group studies. Neuroimage 46:1004-1017.

Tom SM, Fox CR, Trepel C, Poldrack RA (2007) The neural basis of loss aversion in decision-making under risk. Science 315:515-518.

Usher M, McClelland JL (2001) The time course of perceptual choice: the leaky, competing accumulator model. Psychol Rev 108:550592.

Wallis JD (2007) Orbitofrontal cortex and its contribution to decisionmaking. Annu Rev Neurosci 30:31-56.

White V, Webster B, Wakefield M (2008) Do graphic health warning labels have an impact on adolescents' smoking-related beliefs and behaviours? Addiction 103:1562-1571. 